\title{
CIRCULARLY ORDERED DYNAMICAL SYSTEMS
}

\author{
ELI GLASNER AND MICHAEL MEGRELISHVILI
}

\begin{abstract}
We study topological properties of circularly ordered dynamical systems and prove that every such system is representable on a Rosenthal Banach space, hence, is also tame. We derive some consequences for topological groups. We show that several Sturmian like symbolic $\mathbb{Z}^{k}$-systems are circularly ordered. Using some old results we characterize circularly ordered minimal cascades.
\end{abstract}

\section{Contents}

Introduction 1

1. Preliminaries 2

2. C-ordered topological spaces and systems 5

3. Tame families of functions 11

4. Every c-ordered system is Rosenthal representable 12

5. Some Sturmian like symbolic $\mathbb{Z}^{k}$-systems are c-ordered $\quad 15$

6. Minimal c-ordered $\mathbb{Z}$-systems $\quad 17$

References 20

\section{INTRODUCTION}

In this work we study circularly ordered dynamical systems. A circular order (c-order in short) on a set is, intuitively speaking, a linear order which has been bent into a "circle". This concept has its roots at least in an old article of Huntington [24]. It has several applications in Geometry, Combinatorics, Logic, Algebraic Topology and General Topology.

We give some applications of c-order in Topological Dynamics. We say that a dynamical $G$-system $X$ is a c-orderable if $X$, as a topological space, is c-ordered and for each $g \in G$, the corresponding translation $\tilde{g}: X \rightarrow X$ is c-order preserving (Definition 2.8). We investigate the following question:

Question 0.1. Which compact (minimal) dynamical G-systems are c-ordered? In particular, which symbolic $\mathbb{Z}^{k}$-systems are c-ordered?

Note that (in contrast to the circular order case) every linearly ordered minimal $G$-space is trivial. Indeed every compact linearly ordered space has its minimum and maxumum elements, the "end points" of the system, which are necessarily fixed.

As we will see c-orderable dynamical systems are tame. Tame dynamical systems were introduced by A. Köhler [30] and their theory was later developed in a series of works by several authors (see e.g. [14, 16, 18, 23, 28, 15, 44]). Recently, connections to other areas of

Date: August 29, 2016.

2010 Mathematics Subject Classification. Primary 37Bxx, 46-xx; Secondary 54H15, 26A45.

Key words and phrases. circular order, linear order, enveloping semigroup, tame dynamical system, Sturmian system, subshift, symbolic system, Rosenthal space.

This research was supported by a grant of the Israel Science Foundation (ISF 668/13). 
mathematics like Banach spaces, coding theory, substitutions and tilings, and even model theory and logic, were established (see e.g. [2, 25, 7] and the survey [20] for more details).

We recall that a metric dynamical $G$-system $X$ is tame if and only if the enveloping semigroup $E(X)$ has cardinality at most $2^{\aleph_{0}}[16,19]$, iff every element $p \in E(X)$ of its enveloping semigroup is Baire class 1 function $p: X \rightarrow X$ [22]. Other characterizations of tameness are given using the combinatorial notion of independence [28]. Finally, the metrizable tame systems are exactly those systems which admit a representation on a separable Rosenthal Banach space [18] (a Banach space is called Rosenthal if it does not contain an isomorphic copy of $l_{1}$ ). For a survey of this theory we refer the reader to [20].

Using tame families and families of functions with bounded variation we show that (even non-metrizable) circularly ordered dynamical systems are representable on Rosenthal Banach spaces (Theorem 4.5). Rosenthal representable dynamical systems are tame (see [18], or Theorems 3.7 and 3.8 below).

Another approach to obtaining tameness is via the property of being null. For a general group action being null implies being tame. This is a result of Kerr and Li [28] who used independence properties to characterize these classes. In Theorem 2.13 we show easily that c-ordered systems are null, hence tame.

As we show below, many naturally defined symbolic systems, e.g. Sturmian-like systems, are circularly ordered (Theorem 5.3). It thus follows that these systems are tame.

Notation:

(1) As in $[18,21]$ we denote by WRN (respectively, RN) the class of Rosenthal (respectively, Asplund) representable dynamical systems and by Tame the class of tame systems, Definition 1.1. (Recall that a Banach space is Asplund iff its dual has the Radon-Nikodym property.) Within the class of metrizable systems, WRN = Tame.

(2) LODS and CODS will denote the classes of linearly ordered and circularly ordered compact dynamical systems, respectively. Note that LODS $\subset$ CODS.

(3) We will denote by Sturm the class of Sturmian dynamical systems.

Sturm is an important and intensively studied class of binary symbolic systems whose origins go back, at least, to the classical work of Morse and Hedlund [36]. The following inclusions are nontrivial:

Sturm $\subset$ CODS $\subset$ WRN $\subset$ Tame.

Theorems 5.3 and 4.5 yield the first two inclusions. For the inclusion WRN $\subset$ Tame, we refer to $[18,21]$. In particular, LODS $\subset$ WRN. We note that this latter fact gives a valuable information even in the purely topological case (applying it to trivial identity actions), see Section 4.2. We derive also some consequences for topological groups. In particular, Theorem 4.6 shows that for every c-ordered compact space $X$ the topological group $H_{+}(X)$ of all c-order preserving homeomorphisms (equipped with the compact open topology) is Rosenthal representable.

In Section 5 we show that several Sturmian like symbolic $\mathbb{Z}^{k}$-systems are circularly orderable (and minimal). For the case $k=1$ this result gives a new proof of a result by Masui [34]. Multidimensional Sturmian like $\mathbb{Z}^{k}$-systems are studied, e.g., in [4, 12].

Using old results concerning circle homeomorphisms (in particular, Markley's results about Denjoy's minimal systems, [32]) we characterize, in Section 6, infinite circularly ordered metric minimal cascades.

\section{Preliminaries}

We use the notation of $[18,20]$. By a topological space we mean a Tychonoff (completely regular Hausdorff) space. The closure operator in topological spaces will be denoted by cls . 
A function $f: X \rightarrow Y$ is Baire class 1 function if the inverse image $f^{-1}(O)$ of every open set $O$ is $F_{\sigma}$ in $X$. For a pair of topological spaces $X$ and $Y, C(X, Y)$ is the set of continuous functions from $X$ into $Y$. We denote by $C(X)$ the Banach algebra of bounded continuous real valued functions even when $X$ is not necessarily compact.

All semigroups $S$ are assumed to be monoids, i.e., semigroups with a neutral element which will be denoted by $e$. A (left) action of $S$ on a space $X$ is a map $\pi: S \times X \rightarrow X$ such that $\pi(s t, x)=\pi(s, \pi(t, x))$ for every $s, t \in S$ and $x \in X$. We usually simply write $s x$ for $\pi(s, x)$. Also actions are monoidal (meaning $e x=x, \forall x \in X$ ).

An $S$-space is a topological space $X$ equipped with a continuous action $\pi: S \times X \rightarrow X$ of a topological semigroup $S$ on the space $X$. A compact $S$-space $X$ is called a dynamical $S$-system and is denoted by $(S, X)$. Note that in [18] and [19] we deal with the more general case of separately continuous actions. We reserve the symbol $G$ for the case where $S$ is a topological group. As usual, a continuous map $\alpha: X \rightarrow Y$ between two $S$-systems is called an $S$-map or a homomorphism when $\alpha(s x)=s \alpha(x)$ for every $(s, x) \in S \times X$.

For every $S$-space $X$ we have a monoid homomorphism $j: S \rightarrow C(X, X), j(s)=\tilde{s}$, where $\tilde{s}: X \rightarrow X, x \mapsto s x=\pi(s, x)$ is the $s$-translation $(s \in S)$.

The enveloping semigroup $E(S, X)$ (or just $E(X)$ ) for a compact $S$-system is defined as the pointwise closure $\operatorname{cls}(j(S))$ of $\tilde{S}=j(S)$ in $X^{X}$. Then $E(S, X)$ is a compact right topological monoid (i.e. right multiplication $q \mapsto q p, q \in E(S, X)$, is continuous for every $p \in E(S, X))$.

By a cascade on $X$ we mean a $\mathbb{Z}$-action $\mathbb{Z} \times X \rightarrow X$. When dealing with cascades we usually write $(X, \sigma)$, where $\sigma$ is the $s$-translation $X \rightarrow X$ corresponding to $s=1$ ( 0 acts as the identity), instead of $(\mathbb{Z}, X)$.

1.1. Some classes of dynamical systems. We are mainly interested in the class of tame dynamical systems. For the history of this notion we refer to [14] and [18].

Definition 1.1. A compact dynamical $S$-system $X$ is said to be tame if for every $f \in$ $C(X, \mathbb{R})$ the family $f S:=\{f s: s \in S\}$ has no $l_{1}$-subsequence (where, $(f s)(x):=f(s x)$ ); see Definition 3.1. Equivalently, if $f S$ has no independent subsequence (see Theorem 3.6, Definitions 3.1 and 3.4).

The following principal result is a dynamical analog of the Bourgain-Fremlin-Talagrand dichotomy $[3,46]$.

Theorem 1.2. [16] (A dynamical version of BFT dichotomy) Let $X$ be a compact metric dynamical $S$-system and let $E=E(X)$ be its enveloping semigroup. Either

(1) $E$ is a separable Rosenthal compact (hence $E$ is Fréchet and card $E \leq 2^{\aleph_{0}}$ ); or

(2) the compact space $E$ contains a homeomorphic copy of $\beta \mathbb{N}$ (hence card $E=2^{2^{\aleph_{0}}}$ ).

The first possibility holds iff $X$ is a tame $S$-system.

Thus, a metrizable dynamical system is tame iff card $(\mathrm{E}(\mathrm{X})) \leq 2^{\aleph_{0}}$ iff $E(X)$ is a Rosenthal compactum (or a Fréchet space). Moreover, by [22] a metric $S$-system is tame iff every $p \in E(X)$ is a Baire class 1 map $p: X \rightarrow X$. By [18, 19], a general (not necessarily, metrizable) compact $S$-system $X$ is tame iff every $p \in E(X)$ is a fragmented map, Definition 3.2 (equivalently, Baire 1, when $X$ is metrizable). Basic properties and applications of fragmentability in topological dynamics can be found in $[18,20,21]$.

Recall that a dynamical $S$-system $X$ is weakly almost periodic (WAP) if and only if every $p \in E(X)$ is a continuous map. So, every WAP system is tame. The class of hereditarily nonsensitive systems $(\mathrm{HNS})$ is an intermediate class of systems, [20], WAP $\subset$ HNS $\subset$ Tame. A metrizable $S$-system $X$ is $\operatorname{HNS}$ iff $(S, X)$ is RN iff $E(X)$ is metrizable, [16, 22]. The class of tame dynamical systems is quite large. It is closed under subdirect products and factors. 
1.2. Some classes of functions. A compactification of $X$ is a continuous map $\gamma: X \rightarrow Y$ with a dense range where $Y$ is compact. When $X$ and $Y$ are $S$-spaces and $\gamma$ is an $S$-map we say that $\gamma$ is an $S$-compactification.

A function $f \in C(X)$ on an $S$-space $X$ is said to be Right Uniformly Continuous if the induced right action $C(X) \times S \rightarrow C(X)$ is continuous at the point $(f, e)$, where $e$ is the identity of $S$. Notation: $f \in \operatorname{RUC}(X)$. If $X$ is a compact $S$-space then $\operatorname{RUC}(X)=C(X)$. Note that $f \in \operatorname{RUC}(X)$ if and only if there exists an $S$-compactification $\gamma: X \rightarrow Y$ such that $f=\tilde{f} \circ \gamma$ for some $\tilde{f} \in C(Y)$. In this case we say that $f$ comes from the $S$-compactification $\gamma: X \rightarrow Y$.

The function $f$ is said to be: a) WAP; b) Asplund; c) tame if $f$ comes from an $S$ compactification $\gamma: X \rightarrow Y$ such that $(S, Y)$ is: WAP, HNS or tame respectively. For the corresponding classes of functions we use the notation: $\operatorname{WAP}(X), \operatorname{Asp}(X), \operatorname{Tame}(X)$, respectively. Each of these is a norm closed $S$-invariant subalgebra of the $S$-algebra $\operatorname{RUC}(X)$ and $\operatorname{WAP}(X) \subset \operatorname{Asp}(X) \subset \operatorname{Tame}(X)$. For more details see [19, 20]. As a particular case we have defined the algebras $\operatorname{WAP}(S), \operatorname{Asp}(S)$, Tame $(S)$ corresponding to the left action of $S$ on $X:=S$.

1.3. Symbolic systems. The binary Bernoulli shift system is defined as the cascade $(\Omega, \sigma)$, where $\Omega:=\{0,1\}^{\mathbb{Z}}$. We have the natural $\mathbb{Z}$-action on the compact metric space $\Omega$ induced by the $\sigma$-shift:

$$
\mathbb{Z} \times \Omega \rightarrow \Omega, \quad \sigma^{m}\left(\omega_{i}\right)_{i \in \mathbb{Z}}=\left(\omega_{i+m}\right)_{i \in \mathbb{Z}} \quad \forall\left(\omega_{i}\right)_{i \in \mathbb{Z}} \in \Omega, \quad \forall m \in \mathbb{Z} .
$$

More generally, for a discrete group $G$ and a finite alphabet $\Delta$ the compact space $\Delta^{G}$ is a compact $G$-space under the action

$$
G \times \Delta^{G} \rightarrow \Delta^{G}, \quad(s \omega)(t)=\omega(t s), \omega \in \Delta^{G}, \quad s, t \in G .
$$

A closed $G$-invariant subset $X \subset \Delta^{G}$ defines a subsystem $(G, X)$. Such systems are called subshifts or symbolic dynamical systems.

\subsection{Coding functions.}

\section{Definition 1.3.}

(1) Let $G \times X \rightarrow X$ be an action on a (not necessarily compact) space $X, f: X \rightarrow \mathbb{R}$ a bounded (not necessarily continuous) function, and $z \in X$. Define a coding function as follows:

$$
\varphi:=m(f, z): G \rightarrow \mathbb{R}, g \mapsto f(g z) .
$$

(2) When $G=\mathbb{Z}^{k}$ and $f(X)=\{0,1, \ldots, d\}$ we say that $f$ is a $(k, d)$-code. Every such code generates a point transitive subshift $G_{\varphi}$ of $\Delta^{G}$, where $\Delta=\{0,1, \ldots, d\}$ and

$$
G_{\varphi}:=\operatorname{cls}_{p}\{g \varphi: g \in G\} \subset \Delta^{G} \quad(\text { where } g \varphi(t)=\varphi(t g))
$$

is the pointwise closure of the left $G$-orbit $G \varphi$ in the space $\{0,1, \cdots, d\}{ }^{G}$.

(3) In the particular case where $\chi_{D}: X \rightarrow\{0,1\}$ is the characteristic function of a subset $D \subset X$ and $G=\mathbb{Z}$, we get a $(1,1)$-code.

Regarding some dynamical and combinatorial aspects of coding functions see [12, 4].

Question 1.4. When is a coding function $\varphi$ tame? Equivalently, when is the associated transitive subshift system $G_{\varphi} \subset\{0,1\}^{\mathbb{Z}}$, with $\varphi=m\left(D, x_{0}\right)$ tame? When are such subshifts $c$-ordered?

\section{Remarks 1.5.}

(1) Some restrictions on $D$ are really necessary because every binary bisequence $\varphi: \mathbb{Z} \rightarrow\{0,1\}$ can be encoded as $\varphi=m\left(D, x_{0}\right)$. 
(2) It follows from results in [18] that a coding bisequence $c: \mathbb{Z} \rightarrow \mathbb{R}$ is tame iff it can be represented as a generalized matrix coefficient of a Rosenthal Banach space representation. That is, iff there exist: a Rosenthal Banach space $V$, a linear isometry $\sigma \in \operatorname{Iso}(V)$ and two vectors $v \in V, \phi \in V^{*}$ such that

$$
c_{n}=\left\langle\sigma^{n}(v), \varphi\right\rangle=\phi\left(\sigma^{n}(v)\right) \quad \forall n \in \mathbb{Z} .
$$

Recall (see for example [4]) that a bisequence $\mathbb{Z} \rightarrow\{0,1\}$ is Sturmian if it is recurrent and has the minimal complexity $p(n)=n+1$.

Definition 1.6. Let $P_{0}$ be the set $[0, t)$ and $P_{1}$ the set $[t, 1)$; let $z$ be a point in $[0,1)$ (identified with $\mathbb{T}$ ) via the rotation $R_{\alpha}$ we get the binary bisequence

$$
\varphi: \mathbb{Z} \rightarrow\{0,1\}, n \mapsto \varphi(n)=s_{n},
$$

by $s_{n}=0$ when $R_{\alpha}^{n}(z) \in P_{0}, s_{n}=1$ otherwise. Equivalently $\varphi$ is defined as $m\left(\chi_{D}, z\right)$ for $D:=[t, 1)$. These are called Sturmian like codings. With $D=[1-\alpha, 1)$ we get the classical Sturmian bisequences. About a realization of the corresponding subshift see [16] (and Example 2 below). For example, when $\alpha:=\frac{\sqrt{5}-1}{2}$ and $c=1-\alpha$ the corresponding sequence, computed at $z=0$, is called the Fibonacci bisequence.

Every Sturmian bisequence $\varphi$ induces a minimal symbolic system $\mathbb{Z}_{\varphi} \subset\{0,1\}^{\mathbb{Z}}$, which is said to be a Sturmian dynamical system. Similarly one defines Sturmian like systems.

\section{C-Ordered topological SpaCes AND Systems}

First we give one of the most conventional axiomatic for the concept of circular ordering.

Definition 2.1. [29,5] Let $X$ be a set. A ternary relation $R \subset X^{3}$ on $X$ is said to be a circular (or, sometimes, cyclic) order if the following four conditions are satisfied. It is convenient sometimes to write shortly $[a, b, c]$ instead of $(a, b, c) \in R$.

(1) Cyclicity: $[a, b, c] \Rightarrow[b, c, a]$;

(2) Asymmetry: $[a, b, c] \Rightarrow(a, c, b) \notin R$;

(3) Transitivity: $\left\{\begin{array}{l}{[a, b, c]} \\ {[a, c, d]}\end{array} \quad \Rightarrow[a, b, d]\right.$;

(4) Totality: if $a, b, c \in X$ are distinct, then $[a, b, c] \vee[a, c, b]$.

Lemma 2.2. For every c-order on $X$ we have:

(1) $[a, b, c]$ implies that $a, b, c$ are distinct.

(2) $\left\{\begin{array}{l}{[c, a, x]} \\ {[c, x, b]}\end{array} \quad \Rightarrow[a, x, b]\right.$.

For distinct $a, b \in X$ define the (oriented) intervals:

$$
(a, b)_{R}:=\left\{x \in X:[a, x, b], \quad[a, b]_{R}:=(a, b) \cup\{a, b\} .\right.
$$

Sometimes we drop the subscript when context is clear.

\section{Proposition 2.3.}

(1) $[29$, p. 6] For every c-order $R$ on $X$ the family of intervals

$$
\left\{(a, b)_{R}: \quad a, b \in X\right\}
$$

forms a base for a topology $\tau_{R}$ on $X$ which we call the interval topology of $R$.

(2) The topology $\tau_{R}$ of every circular order $R$ is Hausdorff. 
The prototypical example of a c-order the usual (counter-clockwise) circular ordering on the circle $\mathbb{T}$. Identify $\mathbb{T}$, as a set, with $[0,1)$ and define a ternary relation $R \subset[0,1)^{3}$ as follows

$$
(x, y, z) \in R \Leftrightarrow(y-x)(z-y)(z-x)>0 .
$$

Its topology $\tau_{R}$ gives the usual topology on $\mathbb{T}$.

By a linear order $<$ on $X$ we mean a transitive relation which is totally ordered, meaning that for distinct $a, b \in X$ we have exactly one of the alternatives: $a<b$ or $b<a$. As usual, $a \leq b$ will mean that $a<b \vee a=b$.

For every linearly ordered set $(X,<)$ the rays $(a, \rightarrow),(\leftarrow, b)$, with $a, b \in X$, form a subbase for the standard interval topology $\tau_{<}$on $X$. It is well known that the interval topology is always Hausdorff (and even normal). A topological space is said to be Linearly Ordered Topological Space (LOTS) if its topology is $\tau_{<}$for some linear order $<$. Similarly, a topological space is said to be circularly ordered topological space (COTS) if its topology is $\tau_{R}$ for some circular order $R$.

Remark 2.4. [5, page 35]

(1) Every linear order $<$ on $X$ defines a standard circular order $R_{<}$on $X$ as follows: $[x, y, z]$ iff one of the following conditions is satisfied:

$$
x<y<z, y<z<x, z<x<y .
$$

(2) (cuts) Let $(X, R)$ be a c-ordered set and $z \in X$. For every $z \in X$ the relation

$$
z<_{z} a, \quad a<_{z} b \Leftrightarrow[z, a, b] \quad \forall a \neq b \neq z \neq a
$$

is a linear order on $X$ and $z$ is the least element. This linear order restores the original circular order. Meaning that $R_{<_{z}}=R$.

On the set $\{0,1, \cdots, n-1\}$ consider the standard c-order modulo $n$. Denote this cordered set, as well as its order, simply by $C_{n}$. Every finite c-ordered set with $n$ elements is isomorphic (Definition 2.6) to $C_{n}$.

Definition 2.5. Let $(X, R)$ be a c-ordered set. We say that a vector $\left(x_{1}, x_{2}, \cdots, x_{n}\right) \in X^{n}$ is a cycle in $X$ if it satisfies the following two conditions:

(1) For every $[i, j, k]$ in $C_{n}$ and distinct $x_{i}, x_{j}, x_{k}$ we have $\left[x_{i}, x_{j}, x_{k}\right]$;

(2) $x_{i}=x_{k} \Rightarrow\left(x_{i}=x_{i+1}=\cdots=x_{k-1}=x_{k}\right) \vee\left(x_{k}=x_{k+1}=\cdots=x_{i-1}=x_{i}\right)$.

Injective cycle means that all $x_{i}$ are distinct.

Definition 2.6. Let $\left(X_{1}, R_{1}\right)$ and $\left(X_{2}, R_{2}\right)$ be c-ordered sets. A function $f: X_{1} \rightarrow X_{2}$ is said to be $c$-order preserving, or $C O P$, if $f$ moves every cycle to a cycle. Equivalently, if it satisfies the following two conditions:

(1) For every $[a, b, c]$ in $X$ and distinct $f(a), f(b), f(c)$ we have $[f(a), f(b), f(c)]$;

(2) If $f(a)=f(c)$ then $f$ is constant on one of the closed intervals $[a, c],[c, a]$.

We let $M_{+}\left(X_{1}, X_{2}\right)$ be the collection of c-order preserving maps from $X_{1}$ into $X_{2}$.

$f$ is an isomorphism if, in addition, $f$ is a bijection. Denote by $H_{+}(X)$ the group of all COP isomorphisms $X \rightarrow X$ (necessarily homeomorphisms).

A composition of c-order preserving maps is c-order preserving.

\section{Examples 2.7.}

(1) A function $f: C_{n}=\{1,2, \cdots, n\}: \rightarrow X$ is COP if and only if the corresponding vector $\left(f\left(x_{1}\right), f\left(x_{2}\right), \cdots, f\left(x_{n}\right)\right)$ is a cycle in $X$ (Definition 2.5).

(2) Gluing all points of a given closed interval on a c-ordered set defines a COP map. 
(3) In particular, gluing end-points $a, b$ of a gap interval (i.e., $(a, b)$ or $(b, a)$ is empty) of any c-ordered set induces a COP map.

(4) For example, the projection $q: X(c) \rightarrow X$ (from Proposition 2.10) is COP (e.g., $[0,1] \rightarrow \mathbb{T})$

(5) Every COP map $f: X \rightarrow C_{d+1}$ can be interpreted as a standard coloring function from Definition 5.1.

Definition 2.8. We say that a compact $S$-system $(X, \tau)$ is circularly orderable if there exists a compatible circular order $R$ on $X$ such that $X$ is COTS and every $s$-translation $\tilde{s}: X \rightarrow X$ is COP. We denote by CODS the class of all c-orderable systems. Similarly we have the class LOTS of all linearly ordered compact $S$-systems.

For every linearly (circularly) ordered compact space $X$ and every topological subgroup $G \subset H_{+}(X)$, with its compact open topology, the corresponding action $G \times X \rightarrow X$ defines a linearly (circularly) ordered $G$-system.

Proposition 2.9. LODS $\subset$ CODS and LOTS $\subset$ COTS. More precisely: every compact linearly ordered space (S-system) is a circularly ordered space (S-system) with respect to the canonically associated circular order.

Proof. Let $<$ be a linear order on $X$ such that the interval topology $\tau_{<}$is compact. Consider the canonical circular order (Remark 2.4) $R:=R_{<}$on $X$ and the corresponding topology $\tau_{R}$. Then

$$
\tau_{R} \subseteq \tau_{<}
$$

Indeed, it is enough to show that

$$
(a, b)_{R}:=\{x \in X:[a, x, b]\} \in \tau_{<}
$$

for every distinct $a, b \in X$. We have two cases:

(1) $a<b$. Then $(a, b)_{R}=(a, b)_{<}$.

(2) $b<a$. Then $(a, b)_{R}=(\leftarrow, b) \cup(a, \rightarrow)$.

In each case $(a, b)_{R} \in \tau_{<}$.

On the other hand, $\tau_{R}$ is Hausdorff by Proposition 2.3. Since $\tau_{<}$is compact we get

$$
\tau_{<}=\tau_{R}
$$

Finally, note that if an $s$-translation $X \rightarrow X$ is linear order preserving then it is also c-order preserving.

The compactness of $\tau_{<}$is essential. Indeed, the linearly ordered set $X=[0,1)$ with respect to the c-order topology is in fact the circle. This gives a justification of the standard identification of the sets $\mathbb{T}$ and (c-ordered) $[0,1$ ).

The circle $\mathbb{T}$ is clearly a factor space of a (linearly ordered) closed interval $[a, b]$ after identifying the endpoints. The following result shows that we have a similar situation for any c-ordered compact space.

Proposition 2.10. Let $(X, R)$ be a circularly ordered space. Then for every $c \in X$ there exists a linearly ordered space $X(c):=\left(\left[c^{-}, c^{+}\right],<\right)$such that $X$ is homeomorphic to the factor-space of $X(c)$ identifying the endpoints of $X(c)$. Moreover, the corresponding quotient map $q: X(c) \rightarrow X$ (sometimes denoted by $q_{c}$ ) is closed and c-order preserving. When $X$ is compact then so is $X(c)$.

Proof. Take a point $c \in X$ and consider the cut at $c$ where $c$ becomes the minimal element. Denote it by $c^{-}$. Then we get a linearly ordered set $X$ by declaring $x<y$ whenever 
$(c, x, y) \in R$ (see Remark 2.4). Adding to $X$ a new point $c^{+}$as the greatest element we get a linearly ordered set $X(c)=\left[c^{-}, c^{+}\right]=X \cup\left\{c^{+}\right\}$and a natural onto map

$$
q: X(c) \rightarrow X, q\left(c^{-}\right)=q\left(c^{+}\right)=c, q(x)=x \forall x \in\left(c^{-}, c^{+}\right) .
$$

It is easy to see that $q$ is a closed continuous map (hence, a quotient map) with respect to the interval topologies. Moreover, $q$ is c-order preserving.

Continuity: We have to show that $q$ is continuous at every point $z \in X(c)$. Let $U=$ $(a, b)_{R}$ be an open basic neighborhood of $q(z) \in X$, where $a \neq b$. There are two cases:

1) $q(z) \neq c$.

Then $q(z)=z$. We can suppose that $c \notin U$ ( $\tau_{R}$ is Hausdorff). In this case we have necessarily $[c, a, z],[c, z, b]$. By the transitivity, $[c, a, b]$. This means that $a<_{c} b$. Take $V:=$ $(a, b)_{<_{c}}$, an open interval of $z$ in LOTS $X(c)$. Then $q(V) \subset U$. Indeed, $a<_{c} x<_{c} b$ implies that $[c, a, x],[c, x, b]$. Then $[a, x, b]$ by Lemma 2.2. This means that $q(x)=x \in(a, b)_{R}$.

2) $q(z)=c$.

Then $z=c^{-}$or $z=c^{+}$. We have $[a, c, b]$. Equivalently, $[c, b, a]$. So, $b<_{c} a$. Take

$$
V:=(\leftarrow, b) \cup(a, \rightarrow) .
$$

Then $V$ is a neighborhood of $z$ in the LOTS $X(c)$ and $q(V) \subset U:=(a, b)_{R}$. Indeed:

a) If $x<_{c} b$ then we have $[c, x, b]$. Equivalently, $[b, c, x]$. Since $[a, c, b]=[b, a, c]$ the transitivity axiom implies $[b, a, x]$. So we get $[a, x, b]$.

b) If $a<_{c} x$. Then $[c, a, x]$. Since $[c, b, a]$, by Lemma 2.2 we get $[b, a, x]$. Which is equivalent to $[a, x, b]$.

If $X$ is compact then $X(c):=\left[c^{-}, c^{+}\right]$is compact, as a particular case of $X_{A}$ from Lemma 2.11.2 with the singleton $A:=\{c\}$. In this case the closedness of $q$ is clear because $X$ is Hausdorff (Proposition 2.3). In fact, $q$ is closed in general, even when $X$ is not compact. We omit the details.

Every c-ordered space is a normal space. Indeed, recall that every LOTS is normal and the normality is preserved by closed maps onto Hausdorff spaces. Now Proposition 2.10 finishes the proof.

2.1. Splitting points and a construction of c-ordered spaces. We describe a general method for producing c-ordered $G$-systems.

Lemma 2.11. Let $R$ be a circular order on a set $X$ and $A \subset X$.

(1) There exist a canonically defined circularly ordered set $X_{A}=\operatorname{Split}(X ; A)$ and a continuous c-order preserving onto map $\nu: X_{A} \rightarrow X$ such that the preimage $\nu^{-1}(a)$ of any $a \in A$ consists of exactly two points and $\nu^{-1}(x)$ is a singleton for every $x \in X \backslash A$.

(2) If $X$ is compact then $X_{A}$ is also compact. If, in addition, $A$ is countable and $X$ is metrizable then $X_{A}$ is metrizable.

(3) The c-order on $X_{A}$ is uniquely defined. Let $\gamma: M \rightarrow X$ be a c-ordered preserving map and $A \subset X$ such that the preimage $\gamma^{-1}(a)$ of any $a \in A$ consists of exactly two points and $\gamma^{-1}(x)$ is a singleton for every $x \in X \backslash A$. Then $M$, as a c-ordered set, is canonically isomorphic to $X_{A}$.

(4) If $X$ is a c-ordered $G$-space with discrete group $G$ and $A$ is a $G$-invariant subset of $X$ then:

(a) the original action of $G$ on $X$ induces a natural continuous action on $X_{A}$ such that $\nu: X_{A} \rightarrow X$ is a $G$-map.

(b) An inclusion of $G$-invariant subsets $A_{1} \subset A_{2}$ of $X$ induces a natural continuous onto $G$-map $\eta: X_{A_{2}} \rightarrow X_{A_{1}}$ such that $\nu_{1} \circ \eta=\nu_{2}$. 
(c) Assume, in addition, that every point of $A$ is a limit point of $X$ from both sides. Then $X_{A}$ is a minimal dynamical $G$-system iff $X$ is a minimal G-system.

Proof. (1) $X_{A}$, as a set, is $\left\{a^{+}, a^{-}: a \in A\right\} \cup(X \backslash A)$.

$$
\nu: X_{A} \rightarrow X, \quad a^{ \pm} \mapsto a, x \mapsto x \quad \forall a \in A \forall x \in X \backslash A .
$$

Define a natural circular order on $X_{A}$ by the following two rules:

- $[a, b, c]$ for every $(a, b, c) \in X_{A}^{3}$ where $[\nu(a), \nu(b), \nu(c)]$ in $X$.

- $\left[a^{-}, a^{+}, u\right] \quad\left[a^{+}, u, a^{-}\right] \quad\left[u, a^{-}, a^{+}\right]$for every $a \in A, u \notin\left\{a^{+}, a^{-}\right\}$.

The verification of the following claims are straightforward.

Claim 1: $R_{A}$ is a c-order on the set $X_{A}$.

Claim 2: (The topology of $R_{A}$ ) A (standard) base for the circular topology $\tau\left(R_{A}\right)$ on $X_{A}$ of $R_{A}$ at a point of the form $x \in X \backslash A$, is the collection of sets

$$
\nu^{-1}(u, v), \quad x \in(u, v), u, v \in X .
$$

For $s^{-} \in X_{A}$ a basis will be the collection of sets of the form

$$
\left(u, s^{+}\right)=\left\{s^{-}\right\} \cup \nu^{-1}(u, s) .
$$

For $s^{+} \in X_{A}$ a basis will be the collection of sets of the form

$$
\left(s^{-}, v\right)=\left\{s^{+}\right\} \cup \nu^{-1}(s, v) .
$$

Claim 3: $\nu: X_{A} \rightarrow X$ is a continuous c-order preserving map.

(2) If $X$ is compact then one may apply Alexander's subbase theorem to show that $X_{A}$ is compact. Here we use the standard base of $\tau\left(R_{A}\right)$ constructed in Claim 2 .

(3) Let $\gamma^{-1}(a)=\left\{a_{1}, a_{2}\right\}$ and $\left[a_{1}, a_{2}, u\right]$ for some $u \in M$. Then we claim that necessarily $\left[a_{1}, a_{2}, v\right]$ for every $v \notin\left\{a_{1}, a_{2}\right\}$ (this will imply that the c-order on $M$ is uniquely defined). If not then $\left[a_{2}, a_{1}, v\right]$ for some $v \notin\left\{a_{1}, a_{2}\right\}$. Since $\gamma$ is COP we can suppose that $\gamma(v) \neq \gamma(u)$. Using the cyclicity, $\left[a_{2}, a_{1}, v\right]=\left[a_{1}, v, a_{2}\right]$. Together with $\left[a_{1}, a_{2}, u\right]$ we get by Lemma 2.2 that $\left[v, a_{2}, u\right]$. On the other hand by the Transitivity axiom (for $\left[a_{1}, v, a_{2}\right]$ and $\left[a_{1}, a_{2}, u\right]$ ) we have $\left[a_{1}, v, u\right]$. Since $\gamma: M \rightarrow X$ is $\operatorname{COP}$ (and $\gamma(v), a=\gamma\left(a_{1}\right)=\gamma\left(a_{2}\right), \gamma(u)$ are distinct) we obtain $[\gamma(v), a, \gamma(u)]$ and $[a, \gamma(v), \gamma(u)]$. However, this is impossible by the Asymmetry axiom.

(4) (a) The induced action is given by

$$
G \times X_{A} \rightarrow X_{A}, g\left(s^{+}\right)=(g s)^{+}, g\left(s^{-}\right)=(g s)^{-}, g(x)=g x \forall s \in A, \forall x \notin A .
$$

(b) and (c) are straightforward.

Remarks 2.12.

(1) The $G$-space $X_{A}$ from Lemma 2.11 item (4) sometimes will be denoted by $X_{A}=$ $\operatorname{Split}(X, G ; A)$. In the particular case of the cyclic group $G:=\left\langle R_{\alpha}\right\rangle$ generated by an irrational angle $\alpha$ we use the notation $\operatorname{Split}\left(\mathbb{T}, R_{\alpha} ; A\right)$.

(2) Some examples:

(a) Note that if the subset $A \subset X$ is empty then $X_{A}=X$.

(b) For the c-ordered circle $X:=\mathbb{T}$ with $A=\mathbb{T}$ we get the "double circle" of Ellis, [10], which we denote by $\mathbb{T}_{\mathbb{T}}$.

(c) Another important prototype of Lemma 2.11 is [16, Example 14.10]. It gives a concrete realization of the Sturmian subshift, Definition 1.6 (with $t=1-\alpha$ ). In this case the corresponding cascade is $\mathbb{T}_{A}$ with $A:=\{m \alpha, n(1-\alpha): m, n \in \mathbb{Z}\}$. 
(3) For every c-ordered set $K$ and a linearly ordered set $L$ one may define the so-called c-ordered lexicographic product $\mathbb{T} \times L$. See for example [6] and also Figure 1 below. The 2 -circle $\mathbb{T}_{\mathbb{T}}$ as the c-ordered set is the c-ordered lexicographic product $\mathbb{T} \times\{-,+\}$. Every splitting space $X_{A}$ in Lemma 2.11 is a c-ordered subset of $\mathbb{T} \times\{-,+\}$.

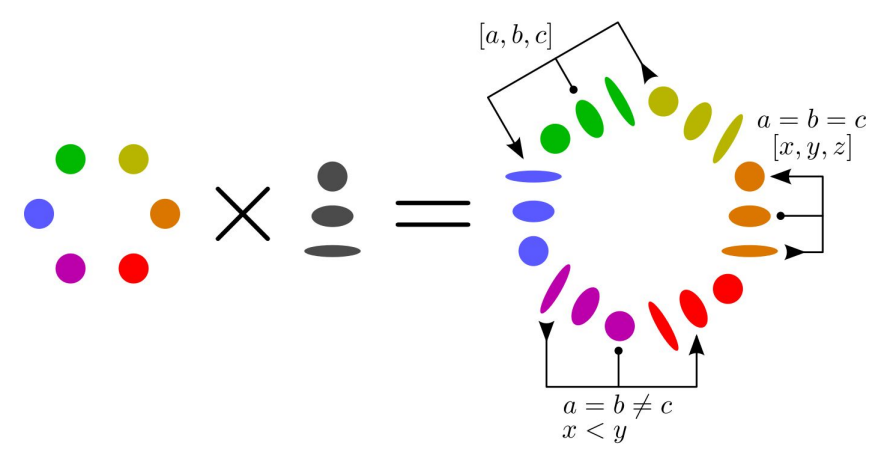

FiguRE 1. c-ordered lexicographic product (from Wikipedia)

(4) The splitting points construction has its roots in linear orders. For every linearly ordered space $X$ and every subset $A \subset X$ one may define a new linearly ordered space $X_{A}$ and a continuous order preserving onto map $X_{A} \rightarrow X$. In particular, we can take $A=X$. Then one gets a generalization of the double arrow space. See, for example, [1]. For $X=A=[0,1]$ the corresponding doubling procedure gives $[0,1] \times\{0,1\}$ with the lexicographic linear order. Removing two isolated points $0^{-}$ and $1^{+}$we get the classical double arrow space which is $X_{A}$ with $X=[0,1]$ and $A=(0,1)$. Note that the "double circle" $\mathbb{T}_{\mathbb{T}}$ is homeomorphic to the double arrow. This space $\mathbb{T}_{\mathbb{T}}$, topologically, is embedded into the enveloping semigroup $E(X)$ of a Sturmian cascade generated by an irrational rotation $T: X \rightarrow X$ as in Definition 1.6. In fact, $\mathbb{T}_{\mathbb{T}}=E(X) \backslash\left\{\sigma^{n}: n \in \mathbb{Z}\right\}$. Moreover, in this case $E(X)$ is c-ordered and the embedding $\mathbb{T}_{\mathbb{T}} \hookrightarrow E(X)$ is c-order preserving. See Section 6.1.

2.2. C-ordered systems are null, hence also tame. We begin by recalling the definition of topological sequence entropy for a general dynamical system $(G, X)$. Let $A=\left\{a_{0}, a_{1}, \ldots\right\}$ be a sequence of of elements of $G$. Given an open cover $\mathcal{U}$, let $N(\mathcal{U})$ be the minimal cardinality of a subcover of $\mathcal{U}$. Define

$$
h_{\text {top }}^{A}(X, \mathcal{U})=\limsup _{n \rightarrow \infty} \frac{1}{n} \log N\left(\bigvee_{i=0}^{n-1} a_{i}{ }^{-1}(\mathcal{U})\right)
$$

The topological entropy along the sequence $A$ is then defined by

$$
h_{\text {top }}^{A}(G, X)=\sup \left\{h_{\text {top }}^{A}(X, \mathcal{U}): \mathcal{U} \text { an open cover of } X\right\} \text {. }
$$

A dynamical system $(G, X)$ is called null if $h_{\text {top }}^{A}(G, X)=0$ for every sequence $A \subset G$.

By results of Kerr and $\mathrm{Li}$ [27, 28] every null system is tame.

Theorem 2.13. A c-ordered metrizable cascade $(G, X)$ is null, hence also tame. 
Proof. It is easy to see that with no loss of generality we can work with open covers $\mathcal{U}$ consisting of a finite collection of open intervals. Let $\mathcal{U}$ be such a cover of cardinality $k$. Now clearly, given $A=\left\{a_{0}<a_{1}<\ldots\right\}$, and denoting $N_{n}=N\left(\bigvee_{i=0}^{n-1} a_{i}^{-1}(\mathcal{U})\right)$, we have

$$
N_{n+1}=N\left(\bigvee_{i=0}^{n} a_{i}^{-1}(\mathcal{U})\right)=N\left(a_{i}^{-1} \mathcal{U} \vee \bigvee_{i=0}^{n-1} a_{i}^{-1}(\mathcal{U})\right) \leq N_{n}+2 k
$$

Therefore, $N_{n} \leq 2 k n$, whence

$$
h_{\text {top }}^{A}(X, \mathcal{U})=\limsup _{n \rightarrow \infty} \frac{1}{n} \log N_{n}=0 .
$$

\section{TAME FAMilies of FUnCtions}

\subsection{Representations on Rosenthal spaces.}

Definition 3.1. Let $f_{n}: X \rightarrow \mathbb{R}$ be a uniformly bounded sequence of functions on a set $X$. Following Rosenthal we say that this sequence is an $l_{1}$-sequence on $X$ if there exists a real constant $a>0$ such that for all $n \in \mathbb{N}$ and choices of real scalars $c_{1}, \ldots, c_{n}$ we have

$$
a \cdot \sum_{i=1}^{n}\left|c_{i}\right| \leq\left\|\sum_{i=1}^{n} c_{i} f_{i}\right\|
$$

For every $l_{1}$-sequence $f_{n}$ its closed linear span in $l_{\infty}(X)$ is linearly homeomorphic to the Banach space $l_{1}$. In fact, the map

$$
l_{1} \rightarrow l_{\infty}(X), \quad\left(c_{n}\right) \rightarrow \sum_{n \in \mathbb{N}} c_{n} f_{n}
$$

is a linear homeomorphic embedding.

A Banach space $V$ is said to be Rosenthal if it does not contain an isomorphic copy of $l_{1}$. Every Asplund (in particular, every reflexive) space is Rosenthal. Recall that a dynamical system $(S, X)$ is WRN means that it is representable on a Rosenthal Banach space.

Definition 3.2. Let $X$ be a topological space and $(Y, \mu)$ is a uniform space. We say that a function $f: X \rightarrow(Y, \mu)$ is fragmented, if for every $\varepsilon \in \mu$ and every nonempty closed subset $A$ of $X$, there exists a non-void relatively open subset $O \subset A$ such that $f(O)$ is $\varepsilon$-small.

Write $\mathcal{F}(X)$ for the collection of real valued fragmented functions on $X$. We note that when $X$ is a compact metrizable space, $f: X \rightarrow \mathbb{R}$ is fragmented iff it is of Baire class 1 iff $f$ is a pointwise limit of a sequence of continuous functions.

Definition 3.3. [18] Let $X$ be a topological space. We say that a subset $F \subset C(X)$ is a Rosenthal family (for $X$ ) if $F$ is norm bounded and the pointwise closure $\operatorname{cls}_{p}(F)$ of $F$ in $\mathbb{R}^{X}$ consists of fragmented maps, that is, $\operatorname{cls}_{p}(F) \subset \mathcal{F}(X)$.

Definition 3.4. A sequence $f_{n}$ of real valued functions on a set $X$ is said to be independent if there exist real numbers $a<b$ such that

$$
\bigcap_{n \in P} f_{n}^{-1}(-\infty, a) \cap \bigcap_{n \in M} f_{n}^{-1}(b, \infty) \neq \emptyset
$$

for all finite disjoint subsets $P, M$ of $\mathbb{N}$.

Definition 3.5. [21] We say that a bounded family $F$ of real valued (not necessarily, continuous) functions on a set $X$ is tame if $F$ does not contain an independent sequence. 
The following useful result combines some known facts. It is based on results of Rosenthal [43], Talagrand [45, Theorem 14.1.7] and van Dulst [9].

Theorem 3.6. Let $X$ be a compact space and $F \subset C(X)$ a bounded subset. The following conditions are equivalent:

(1) $F$ is a tame family.

(2) $F$ does not contain a subsequence equivalent to the unit basis of $l_{1}$.

(3) $F$ is a Rosenthal family for $X$.

Theorem 3.7. Let $X$ be a compact $S$-space. Suppose there is a point separating bounded $S$-invariant family $F$ of continuous real valued functions on $X$ such that $F$ is a tame family. Then $(S, X)$ is a tame system.

Proof. Let $p$ be an arbitrary element of the enveloping semigroup $E(S, X)$. Let $\mathcal{F}=\operatorname{cls} F$ be the pointwise closure of $F$ in $\mathbb{R}^{X}$. Then every element of $\mathcal{F}$ is fragmented by Theorem 3.6. Clearly $\{f \circ p: f \in F\} \subset \mathcal{F}$. As $F$ separates points on $X$ this, in turn, easily implies (see, [18, Lemma 2.3.3]) that $p$ is a fragmented map. Thus $(S, X)$ is tame by a characterization of tame systems mentioned in Section 1.1.

In fact, we have the following sharper statement (see [18, Theorem 6.5], and with more details [21, Theorem 3.12]):

Theorem 3.8. Let $X$ be a compact $S$-space. The following conditions are equivalent:

(1) $(S, X)$ is WRN, that is, Rosenthal representable.

(2) there exists a point separating bounded $S$-invariant family $F$ of continuous real functions on $X$ such that $F$ is a tame family (equivalently, Rosenthal family).

\section{Every C-ORDERED SYStem is Rosenthal REPRESENTABle}

\subsection{Families of functions with bounded variation are tame.}

\section{Definition 4.1.}

(1) Let $(X,<)$ be a linearly ordered set and $(M, d)$ is a metric space. We say that a bounded function $f:(X,<) \rightarrow(M, d)$ has variation not greater than $r$ (notation: $\left.f \in B V_{r}\right)$ if

$$
\sum_{i=1}^{n-1} d\left(f\left(x_{i}\right), f\left(x_{i+1}\right)\right) \leq r
$$

for every choice of $x_{1} \leq x_{2} \leq \cdots \leq x_{n}$ in $X$.

(2) For circularly ordered sets $(X, R)$ (instead of $(X,<))$ the definition is similar but we take cycles $x_{1}, x_{2}, \cdots, x_{n}$ in $X$ (Definition 2.5) and require that

$$
\sum_{i=1}^{n} d\left(f\left(x_{i}\right), f\left(x_{i+1}\right)\right) \leq r
$$

where $x_{n+1}=x_{1}$.

The least upper bound of all such possible sums is the variation of $f$; notation (both cases): $\Upsilon(f)$. If $\Upsilon(f) \leq r$ then we write $f \in B V_{r}(X, M)$ or, simply $B V_{r}(X)$ if $(M, d)=\mathbb{R}$. If $f(X) \subset[c, d]$ for some reals $c \leq d$ then we write also $f \in B V_{r}(X,[c, d])$.

Remarks 4.2. 
(1) In the case of a linearly ordered set $(X,<)$ denote by $M_{+}(X,[c, d])$ the set of all order-preserving functions $X \rightarrow[c, d]$. Then $M_{+}(X,[c, d]) \subset B V_{r}(X,[c, d])$ for every $r \geq d-c$. In particular, $M_{+}(X,[0,1]) \subset B V_{1}(X,[0,1])$.

(2) For every finite interval partition of a c-ordered set $(X, R)$, every finite coloring $f: X \rightarrow \Delta \subset \mathbb{R}$ of this partition is a function with bounded variation.

(3) Note that the sum in Equation 4.2 remains the same under the standard cyclic translation $(+1(\bmod n))$. Also, one may reduce the computations in Definition 4.1.2 to the injective cycles.

(4) Let $q: X(c) \rightarrow X$ be the natural quotient (from Proposition 2.10) for some $c \in X$, where $X(c)$ carries the natural linear order, and let $f: X(c) \rightarrow M, f_{0}: X \rightarrow M$ be functions such that $f=f_{0} \circ q$. Then (using Remark 4.2.3) we have

$$
\Upsilon(f) \leq \Upsilon\left(f_{0}\right) \leq \Upsilon(f)+\operatorname{diam} M
$$

(5) For every COP map $f_{1}: X \rightarrow Y$ and every $f_{2} \in B V_{r}(Y, M)$ we have $f_{2} \circ f_{1} \in$ $B V_{r}(X, M)$.

Lemma 4.3. [35] For every linearly ordered set $(X,<)$ the set $B V_{r}(X,[c, d])$ is a tame family of functions. In particular, this is true also for $M_{+}(X,[c, d])$.

Let $\left(\mathbb{T}, R_{\alpha}\right)$ be the cascade generated by an irrational rotation $R_{\alpha}$ of the circle $\mathbb{T}$. Let $f:=$ $\chi_{D}: \mathbb{T} \rightarrow\{0,1\}$ be the (discontinuous) characteristic function of the arc $D=[a, a+s) \subset \mathbb{T}$. Consider the $\mathbb{Z}$-orbit $F$ of this function induced by the cascade $\left(\mathbb{T}, R_{\alpha}\right)$. Then $F$ is a tame family of (discontinuous) functions on $\mathbb{T}$. Much more generally we have:

Theorem 4.4. Let $(X, R)$ be a c-ordered set. Then any family of functions $\left\{f_{i}: X \rightarrow\right.$ $[c, d]\}_{i \in I}$ with finite total variation is tame.

Proof. It suffices to show that $B V_{r}(X,[c, d])$ is tame for every $r>0$. The case of a c-ordered $(X, R)$ can be reduced to the linearly ordered cut space $X(c)$, where we have Lemma 4.3, using Remark 4.2.4 and the observation that the family $\left\{f_{i}\right\}$ is tame iff so is $\left\{f_{i} \circ q\right\}$.

We are now ready to prove:

Theorem 4.5. Every c-ordered compact, not necessarily metrizable, $S$-space $X$ is Rosenthal representable (that is, WRN), hence, in particular, tame. So, CODS $\subset \mathrm{WRN} \subset$ Tame.

Proof. Let $X$ be a c-ordered compact $S$-system. We have to show that the $S$-system $X$ is WRN. By Theorem 3.8, this is equivalent to showing that there exists a point separating bounded $S$-invariant family $F$ of continuous real valued functions on $X$ such that $F$ is tame. By Theorem 4.4 bounded total variation of $F$ is a sufficient condition for its tameness.

Let $a \neq b$ in $X$. We can assume that $X$ is infinite. Take some third point $c \in X$. As in Proposition 2.10 consider the cut at $c$ where $c$ becomes the minimal element. We get a compact linearly ordered set $X(c)=\left[c^{-}, c^{+}\right]$and a natural quotient map

$$
q: X(c) \rightarrow X, q\left(c^{-}\right)=q\left(c^{+}\right)=c
$$

and $q(x)=x$ in other points.

We have two similar cases:

1) $c^{-}<a<b<c^{+}$

2) $c^{-}<b<a<c^{+}$

We explain the proof only for the first case.

Since $X(c)=\left[c^{-}, a\right] \cup[a, b] \cup\left[b, c^{+}\right]$is a linearly ordered compact space, its closed intervals $[a, b]$ and $\left[b, c^{+}\right]$are also compact LOTS. By Nachbin's results [37] continuous linear order 
preserving maps from compact LOTS to $[0,1]$ separate the points. Therefore, one may choose continuous maps

$$
f_{1}:\left[c^{-}, a\right] \rightarrow[0,1], f_{2}:[a, b] \rightarrow[0,1], \quad f_{3}:\left[b, c^{+}\right] \rightarrow[0,1]
$$

such that $f_{1}$ is identically zero, $f_{2}$ and $f_{3}$ are order preserving, and

$$
f_{2}(b)=f_{3}(b)=1, f_{2}(a)=f_{3}\left(c^{+}\right)=0 .
$$

These three functions define a continuous function $f:\left[c^{-}, c^{+}\right] \rightarrow[0,1]$. It is easy to see that $f$ has total variation not greater than 2, that is, $f \in B V_{2}(X(c))$ and, clearly, $0=f(a) \neq f(b)=1$.

The factor-function $f_{0}: X \rightarrow[0,1]$ (with $q\left(f_{0}(x)\right.$ ) $=f(x)$ ) is continuous because $q$ : $X(c) \rightarrow X$ is a quotient map and $f$ is continuous. Moreover, by Remark 4.2.4 we have

$$
\Upsilon(f) \leq \Upsilon\left(f_{0}\right) \leq \Upsilon(f)+1
$$

Thus, $\Upsilon\left(f_{0}\right) \leq 3$. Then, $\Upsilon\left(f_{0} s\right) \leq 3$ (by Remark 4.2.5) for every $s \in S$, because every $s$ translation preserves the c-order. Define $F:=F_{0} S$, where $F_{0}$ is a set of continuous functions $X \rightarrow[0,1]$ with variation $\leq 3$. Since $F_{0}$ separates the points of $X, F_{0} S$ is the desired bounded point-separating family of continuous functions which is tame and $S$-invariant. Now apply Theorem 3.8.

4.2. Some purely topological notes. As a direct topological consequence of Theorem 4.5 note that every compact COTS is WRN. For instance, the two arrows space $K$ is Rosenthal representable. At the same time, $K$ is not Asplund representable (that is not RN) by a result of Namioka [38, Example 5.9]. That is, $K \in \mathrm{WRN} \backslash \mathrm{RN}$.

In a recent paper [33] Martinez-Cervantes shows that a continuous image of a WRN compact space need not be WRN. This answers a question from [21]. Note that $\beta \mathbb{N}$ is not WRN, a result of Todorčević (see [21]). Another result from [33], shows that the Talagrand's compact is also not WRN.

\subsection{Representations of topological groups on Rosenthal spaces.}

Theorem 4.6. Topological group $H_{+}(X)$ (with compact open topology) is Rosenthal representable for every c-ordered compact space $X$. For example, it is true for $H_{+}(\mathbb{T})$.

Proof. (See also [21]) Let $G:=H_{+}(X)$ with its compact open topology. The dynamical $G$-system $X$ admits a representation $(h, \alpha)$

$$
h: G \rightarrow \operatorname{Iso}(V), \quad \alpha: X \rightarrow B^{*}
$$

on a Rosenthal Banach space $V$ by Theorem 4.5. Then the homomorphism

$$
h^{*}: G \rightarrow \operatorname{Iso}(V), \quad g \mapsto h\left(g^{-1}\right)
$$

is a topological group embedding because the strong operator topology on Iso $(V)^{o p}$ is identical with the compact open topology inherited from the action of this group on the weak-star compact unit ball $\left(B^{*}, w^{*}\right)$ in the dual $V^{*}$.

The Ellis compactification $j: G \rightarrow E(G, \mathbb{T})$ of the Polish group $G=H_{+}(\mathbb{T})$ is a topological embedding. In fact, observe that the compact open topology on $j(G) \subset C_{+}(\mathbb{T}, \mathbb{T})$ coincides with the pointwise topology. This observation implies, by [20, Remark 4.14] that Tame $(G)$ separates points and closed subsets.

Although $G$ is representable on a (separable) Rosenthal Banach space, we have $\operatorname{Asp}(G)=$ $\{$ constants\} and therefore any Asplund representation of this group is trivial (this situation is similar to the case of the group $\left.H_{+}[0,1],[17]\right)$. Indeed, we have $\operatorname{SUC}(G)=\{$ constants $\}$ by [17, Corollary 11.6] for $G=H_{+}(\mathbb{T})$, and we recall that for every topological group $\operatorname{Asp}(G) \subset \operatorname{SUC}(G)$. 
Theorem 4.6 suggests the following:

Definition 4.7. Let us say that a topological group $G$ is c-orderly if $G$ is a topological subgroup of $H_{+}(X)$ for some circularly ordered compact space $X$.

Thus, every orderly topological group $G$ is Rosenthal representable. Recall that it is unknown yet (see [19, 20]) whether every Polish group is Rosenthal representable.

Remark 4.8. If $H<G$ is a subgroup of finite index and $(\mathrm{G}, \mathrm{X})$ is a dynamical system, then clearly $F E(H, X)=E(G, X)$, with $F$ a finite set of representatives of cosets of $H$ in $G$. Thus $(G, X)$ is tame iff $(H, X)$ is tame. In particular, we see that the action of $H_{ \pm}(\mathbb{T})=H(\mathbb{T})$ on the circle $\mathbb{T}$ is tame. In fact, $H_{ \pm}(X)=H(X)$ for every connected c-ordered compact space $X$, where $H_{ \pm}(X)$ is the group of all bijections $h: X \rightarrow X$ which are either c-order preserving or c-order reversing. This follows from [29, Theorem 14]

4.4. When the universal system $M(G)$ is c-ordered ? Recall that for every topological group $G$ there exists, the canonically defined, universal minimal system $M(G)$ and universal irreducible affine $G$-system $I A(G)$. See for example, [10, 13, 41, 47, 26]. In [21] we discuss some examples of Polish groups $G$, for which $M(G)$ and $I A(G)$ are tame. These properties can be viewed as natural generalizations of extreme amenability and amenability, respectively.

Question 4.9. Find examples where $M(G)$ is c-ordered (more generally, tame).

Let us say that $G$ is intrinsically c-ordered (intrinsically tame) if the $G$-system $M(G)$ is c-ordered (respectively, tame). In particular, we see that $G=H_{+}(\mathbb{T})$ is intrinsically cordered, using a well known result of Pestov [40] which identifies $M(G)$ as the tautological action of $G$ on the circle $\mathbb{T}$. Note also that the Polish groups Aut $(\mathbf{S}(2))$ and $\mathrm{A} u t(\mathbf{S}(3))$, of automorphisms of the circular directed graphs $\mathbf{S}(2)$ and $\mathbf{S}(3)$, are also intrinsically cordered. The universal minimal $G$-systems for the groups Aut $(\mathbf{S}(2))$ and Aut $(\mathbf{S}(3))$ are computed in [48]. One can show that $M(G)$ for these groups are c-ordered, see [21].

\section{Some Sturmian LiKe Symbolic $\mathbb{Z}^{k}$-Systems ARE C-ORDERED}

We will see that several coding functions come from c-ordered systems (in particular, are tame) including some multidimensional analogues of Sturmian sequences. The latter are defined on the groups $\mathbb{Z}^{k}$ and instead of the characteristic function $f:=\chi_{D}$ (with $D=[0, c)$ ) we consider finite coloring of the space leading to shifts with finite alphabet.

Definition 5.1. Let $X$ be a c-ordered set and $c_{0}, c_{1}, \ldots, c_{d}$ be a cycle of distinct elements. Consider a finite cyclic partition

$$
X=\cup_{i=0}^{d}\left[c_{i}, c_{i+1}\right),
$$

where $c_{d+1}=c_{0}$. We say that $f: X \rightarrow \Delta:=\{0,1, \ldots, d\}$ is a coloring function if $f$ is constant on each arc $\left[c_{i}, c_{i+1}\right)$. If, in addition, different arcs have different colors (equivalently, $f$ is onto) we say that $f$ is proper. For instance, the following standard coloring function is proper (and a COP map with $\Delta=C_{d+1}$ ).

$$
f: X \rightarrow \Delta:=\{0, \ldots, d\}, \quad f(t)=i \text { iff } t \in\left[c_{i}, c_{i+1}\right) .
$$

Definition 5.2. For $X:=\mathbb{T}$ consider a cyclic partition $\mathbb{T}=\cup_{i=0}^{d}\left[c_{i}, c_{i+1}\right)$ and a proper coloring function $f: \mathbb{T} \rightarrow \Delta$. For a given $k$-tuple $\left(\alpha_{1}, \ldots, \alpha_{k}\right) \in \mathbb{T}^{k}$ of angles, where at least one of them is irrational, define the homomorphism

$$
h: \mathbb{Z}^{k} \rightarrow \mathbb{T}, \quad s:=\left(n_{1}, \ldots, n_{k}\right) \mapsto n_{1} \alpha_{1}+\cdots+n_{k} \alpha_{k},
$$


and the induced c-order preserving action $\mathbb{Z}^{k} \times \mathbb{T} \rightarrow \mathbb{T},(s, t) \mapsto t+h(s)$. Given a point $z \in \mathbb{T}$ consider the corresponding coding function

$$
\varphi=m(f, z): \mathbb{Z}^{k} \rightarrow\{0, \ldots, d\} \quad\left(n_{1}, \ldots, n_{k}\right) \mapsto f\left(z+n_{1} \alpha_{1}+\cdots+n_{k} \alpha_{k}\right) .
$$

We call such a sequence a multidimensional $(k, d)$-Sturmian like sequence.

The case of $k=1$ is studied in [39, 2]. For some multidimensional Sturmian like $\mathbb{Z}^{k}$ systems see, for example, [4, 12]. Consider the associated symbolic Sturmian like $\mathbb{Z}^{k}$-system $G_{\varphi} \subset \Delta^{\mathbb{Z}^{k}}$ (from Definition 1.3). The following theorem yields many examples of c-ordered (in particular, tame) symbolic $\mathbb{Z}^{k}$-systems and tame coding functions.

Theorem 5.3. Let $\varphi=m(f, z)$ be the coding function induced by a proper coloring function $f: \mathbb{T} \rightarrow \Delta$. Then the corresponding symbolic Sturmian like $\mathbb{Z}^{k}$-system $G_{\varphi} \subset\{0,1, \cdots, d\} \mathbb{Z}^{k}$ is isomorphic to a c-ordered minimal metric $\mathbb{Z}^{k}$-system $\mathbb{T}_{A}$ from Lemma 2.11 for some $A \subset \mathbb{T}$. Furthermore, $G_{\varphi}$ is a tame $\mathbb{Z}^{k}$-system and $\varphi \in \operatorname{Tame}\left(\mathbb{Z}^{k}\right)$.

Proof. First observe that the action of $G:=\mathbb{Z}^{k}$ on $\mathbb{T}$

$$
G \times \mathbb{T} \rightarrow \mathbb{T}, \quad\left(\left(n_{1}, \ldots, n_{k}\right), t\right) \mapsto t+n_{1} \alpha_{1}+\cdots+n_{k} \alpha_{k} .
$$

is c-ordered. By our assumption one of the the angles $\alpha_{1}, \ldots, \alpha_{k} \in[0,1)$ is irrational, say $\alpha_{1}$. Thus, the image $h(G)$ is dense in $\mathbb{T}$ and the action $G \times \mathbb{T} \rightarrow \mathbb{T}$ is minimal.

Let $E$ be the set of all endpoints $c_{i}$ of the given arcs from Definition 5.2. Let $A:=G E$. Consider the splitting points construction. By Lemma 2.11 we get a compact metrizable minimal c-ordered space $\mathbb{T}_{A}$, the $G$-projection $\nu: \mathbb{T}_{A} \rightarrow \mathbb{T}$, and the continuous action

$$
G \times \mathbb{T}_{A} \rightarrow \mathbb{T}_{A}, g\left(s^{+}\right)=(g s)^{+}, g\left(s^{-}\right)=(g s)^{-}, g(x)=g x \forall s \in A, \forall x \notin A .
$$

As in Definition 5.2 consider the coding function

$$
\varphi:=m(f, z): \mathbb{Z}^{k} \rightarrow \mathbb{R}, \quad g \mapsto f(g(z))
$$

and the corresponding subshift (Definition 1.3.2)

$$
G_{\varphi}:=\operatorname{cls}_{p}(G \varphi) \subset\{0,1, \cdots, d\}^{\mathbb{Z}^{k}} .
$$

Then $G_{\varphi}$ is a symbolic $\mathbb{Z}^{k}$-system and $\gamma_{1}: G \rightarrow G_{\varphi}, g \mapsto g \varphi$ is a $G$-compactification.

Consider also the $G$-compactification $\gamma_{2}: G \rightarrow \mathbb{T}_{A}$, where $\gamma_{2}$ is the (dense) orbit $G$ map $\gamma_{2}(g)=g\left(z^{+}\right)$, if $z \in A$ and $\gamma_{2}(g)=g(z)$, if $z \notin A$. Our aim is to show that these $G$-compactifications $\gamma_{1}$ and $\gamma_{2}$ are $G$-isomorphic.

$G_{\varphi} \subset\{0,1, \cdots, d\}^{\mathbb{Z}^{k}}$ is a pointwise compact subset of $C(G)$. Consider the (pointwise continuous) function $\widetilde{\varphi}: G_{\varphi} \rightarrow \mathbb{R}, \omega \mapsto \omega(e)$. Then $\varphi=\widetilde{\varphi} \circ \gamma_{1}$. Clearly, $\widetilde{\varphi} G$ separates points of $G_{\varphi}$. These facts easily yield (see also [16, Proposition 2.4]) that the $G$-subalgebra $\mathcal{A}_{1} \subset \operatorname{RUC}(G)$ of the $G$-compactification $\gamma_{1}: G \rightarrow G_{\varphi}$ is the least Banach unital $G$ subalgebra in $\operatorname{RUC}(G)$ (with respect to the right action of $G$ ) which contains $\varphi G$.

Using the given coloring function $f: \mathbb{T} \rightarrow \Delta$ define

$$
f^{+}: \mathbb{T}_{A} \rightarrow \Delta, \quad f^{+}(t)=f(t) \quad \forall t \in\left[c_{i-1}^{+}, c_{i}^{+}\right) .
$$

This function is continuous because each $\left[c_{i}^{+}, c_{i+1}^{+}\right)$is a clopen subset of $\mathbb{T}_{A}$. Note that $\varphi: G \rightarrow \Delta$ comes from $\gamma_{2}: G \rightarrow \mathbb{T}_{A}$. Namely, $\varphi=f^{+} \circ \gamma_{2}$. Therefore, the $G$-subalgebra $\mathcal{A}_{2} \subset \operatorname{RUC}(G)$ of the compactification $\gamma_{2}$ contains $\varphi$ and hence also $\mathcal{A}_{1}$ (by its minimality property mentioned before). So, there exists a quotient $G$-map $q: \mathbb{T}_{A} \rightarrow G_{\varphi}$ such that $q \circ \gamma_{2}=\gamma_{1}$. We have to show that $q$ is isomorphism, equivalently, that we have also the converse inclusion $\mathcal{A}_{2} \subseteq \mathcal{A}_{1}$. By the basic properties of compactifications it suffices to show now that the $G$-orbit $f^{+} G$ of $f^{+}: \mathbb{T}_{A} \rightarrow \mathbb{R}$ separates the points of $\mathbb{T}_{A}$. Let $x, y \in \mathbb{T}_{A}$ be distinct points. Consider the $G$-map $\nu: \mathbb{T}_{A} \rightarrow \mathbb{T}$. We have the following two cases: 
(a) (twins) $\nu(x)=\nu(y)$.

So, $x=c_{i}^{-}, y=c_{i}^{+}$, or $x=c^{+}, y=c^{-}$. Then $f^{+}\left(c_{i}^{-}\right)=f\left(c_{i-1}\right), f^{+}\left(c_{i}^{+}\right)=f\left(c_{i}\right)$ or $f^{+}\left(c_{i}^{+}\right)=f\left(c_{i}\right), f^{+}\left(c_{i}^{-}\right)=f\left(c_{i-1}\right)$. Since $f$ is proper, in both cases we have $f^{+}(x) \neq f^{+}(y)$.

(b) (non-twins) $x_{0}:=\nu(x) \neq y_{0}:=\nu(y)$.

Then since $\alpha_{1}$ is irrational there exists $n \in \mathbb{Z}$ such that the points $x_{0}+n \alpha_{1}$ and $y_{0}+n \alpha_{1}$ belong to different arcs in the given partition. Since $f$ is proper, it follows that the function

$$
f^{+} g: \mathbb{T}_{A} \rightarrow \Delta, t \mapsto f^{+}(g(t))
$$

with $g:=\left(n_{1}, 0, \cdots, 0\right) \in \mathbb{Z}^{k}$ separates the points $x, y$.

So, $\gamma_{1}$ and $\gamma_{2}$ are $G$-isomorphic. In particular, $G_{\varphi}$ is a tame $G$-system (being c-ordered, Theorem 4.5). Finally, $\varphi \in \operatorname{Tame}\left(\mathbb{Z}^{k}\right)$ because $\varphi=\widetilde{\varphi} \circ \gamma_{1}$.

\section{Remarks 5.4.}

(1) In particular, every Strurmian like rotation bisequence (for $k=1, d=1$ ) from Definition 1.6 satisfies the conditions of Theorem 5.3. For the case $k=1$ this result gives a new proof of a result in [34]. One may get in this way c-ordered subshifts $X \subset\{0,1\}^{\mathbb{Z}}$ which are not Sturmian (with complexity greater than $p(n)=n+1$ ).

(2) At least for the case of $k=1$, the tameness of the corresponding symbolic $\mathbb{Z}$-systems coming from coding functions of Definition 5.2 can be proved also by results of Pikula [39] and Aujogue [2].

(3) Theorem 5.3 can be modified for a slightly more general partitions $\mathbb{T}=\cup_{i=0}^{d} I_{i}$, where each $I_{i}$ is an arc on $\mathbb{T}$ (open, closed or containing one of the boundary points).

\section{Minimal C-ORDERed $\mathbb{Z}$-Systems}

Theorem 6.1. Let $(X, \sigma)$ be a circularly ordered topologically transitive cascade with no isolated points. Then $(X, \sigma)$ is minimal and there exists an irrational $\alpha \in \mathbb{R}$ and an $R_{\alpha^{-}}$ invariant subset $A \subset \mathbb{T}=\mathbb{R} / \mathbb{Z}$ such that: $(X, \sigma) \cong \operatorname{Split}\left(\mathbb{T}, R_{\alpha} ; A\right)$ (defined in Lemma 2.11 and Remark 2.12).

Proof. Let $C_{X} \subset X \times X \times X$ be the circular order on $X$. Let

$$
\Omega=\left\{\left(x, x^{\prime}\right) \in X \times X: x=x^{\prime} \text {, or }\left(x, x^{\prime}\right)_{C}=\emptyset \text {, or }\left(x^{\prime}, x\right)_{C}=\emptyset\right\} .
$$

Note that if the pairs $\left(x, x^{\prime}\right)$ and $\left(x^{\prime}, x^{\prime \prime}\right)$ are in $\Omega$ then necessarily $x^{\prime \prime}=x$, as otherwise $x^{\prime}$ would be an isolated point. Now it is easy to check that $\Omega$ is an ICER on $X$.

Let $Y=X / \Omega$ be the quotient dynamical system and let $\pi: X \rightarrow Y$ denote the quotient homomorphism. We denote the corresponding transformation on $Y$ by $S$. Thus $\pi(\sigma x)=$ $S \pi(x)$ for every $x \in X$.

It is easy to see that the circular order on $X$ induces a circular order, $C_{Y}$, on $Y$ and that $\pi$ respects this ordering in the sense of Definition 2.6.

As $Y$ is compact it follows that $C_{Y}$ is a complete and dense circular order. Since $Y$ is a minimal cascade it is in particular separable. This, in turn, implies that the countable collection of open intervals defined via a dense countable subset forms a countable basis for the topology on $Y$. Therefore $Y$ (with its order topology) is a connected metrizable compact space (a continuum). Moreover, it has the property that by omitting any two distinct points it becomes disconnected. These properties characterize the circle $\mathbb{T}=\mathbb{R} / \mathbb{Z}$ (see, for example, [5]).

As $S: Y \rightarrow Y$ is topologically transitive it follows, by a theorem of Poincaré [42], that $S$ is conjugate to an irrational rotation $R_{\alpha}: \mathbb{T} \rightarrow \mathbb{T}$, given by $R_{\alpha}(y)=y+\alpha(\bmod 1)$ for some irrational number $\alpha \in \mathbb{R}$. We now identify $(Y, S)=\left(\mathbb{T}, R_{\alpha}\right)$. 
It follows that $A$ is $R_{\alpha}$-invariant, where $A=\left\{y \in Y:\left|\pi^{-1}(y)\right|=2\right\}$, and $\mathbb{T} \backslash A=\{y \in Y$ : $\left.\left|\pi^{-1}(y)\right|=1\right\}$. In turn, this fact implies that $(X, \sigma)$ is also minimal. Moreover, by Lemma 2.11 .3 we easily get that $(X, \sigma)$ is isomorphic to $\operatorname{Split}\left(\mathbb{T}, R_{\alpha} ; A\right)$.

In [8] Denjoy shows that a circle homeomorphism $\psi: \mathbb{T} \rightarrow \mathbb{T}$ is either minimal (in which case it is conjugate to a minimal rotation), or it admits a unique minimal set $X \subsetneq \mathbb{T}$ which is a Cantor set. In [32] Markley gives a nice characterization of minimal cascades $(X, \sigma)$ which can be embedded into a circle homeomorphism system $(\mathbb{T}, \psi)$.

In the following theorem $\Delta=\{(x, x): x \in X\}$ is the diagonal subset of $X \times X, P \subset X \times X$ is the proximal relation, $P^{\prime}$ is the set of accumulation points of $P$, and $D \subset X \times X$ is the distal structure relation of the system $(X, \sigma)$; i.e. $D$ is the smallest ICER such that $X / D$ is distal (see [11]).

Theorem 6.2 (Markley). Let $(X, \sigma)$ be a minimal cascade, where $X$ is a compact Hausdorff space. Then $(X, \sigma)$ can be imbedded in some $(\mathbb{T}, \psi)$, where $\psi$ has no periodic points, if and only if $P^{\prime} \subset \Delta, P \backslash \Delta$ is countable, and $X / D$ is homeomorphic to $\mathbb{T}$.

It also follows from his proof that when $(X, \sigma)$ satisfies the assumptions of the theorem then $P=D$ and the cardinality of $P[x]$ is one or two for each $x \in X$. Thus the homomorphism $\pi: X \rightarrow X / D=\mathbb{T}$ is either an isomorphism or it is one-to-one on the complement of the preimage of a countable set in $\mathbb{T}$, and two-to-one on the preimage of that set. Every such cascade, being a compact system topologically embeddable in $(\mathbb{T}, \psi)$, inherits a topologically compatible circular order.

Combining these results we get:

Theorem 6.3. Let $(X, \sigma)$ be a minimal cascade with $X$ compact Hausdorff. The following conditions are equivalent.

(1) $(X, \sigma)$ is an infinite, circularly ordered, minimal cascade and $X$ is second countable.

(2) $(X, \sigma)$ can be imbedded in some $(\mathbb{T}, \psi)$, where $\psi$ is a homeomorphism of $\mathbb{T}$ which has no periodic points.

(3) $(X, \sigma)$ satisfies Markley's conditions (namely, $P^{\prime} \subset \Delta, P \backslash \Delta$ is countable, and $X / D$ is homeomorphic to $\mathbb{T}$ ).

(4) There exists an irrational number $\alpha \in \mathbb{R}$ such that $(X, \sigma) \cong \operatorname{Split}\left(\mathbb{T}, R_{\alpha} ; A\right)$ for some countable, $R_{\alpha}$-invariant subset $A \subset \mathbb{T}$.

Proof. Markley's theorem asserts that (2) and (3) are equivalent. From his proof it follows that (3) implies (4). It follows from Theorem 6.1 that (1) and (4) are equivalent. Finally, interpolating an interval between any pair of the countable collection $\left\{\left(a^{+}, a^{-}\right): a \in A\right\}$ of split points in $\operatorname{Split}\left(\mathbb{T}, R_{\alpha} ; A\right)$, it is easy to see that the resulting space is a circle $\mathbb{T}$. Then one can define a homeomorphism $\psi: \mathbb{T} \rightarrow \mathbb{T}$, in such a way that the corresponding inclusion map $i: X \rightarrow \mathbb{T}$ is an embedding of dynamical systems $i:(X, \sigma) \rightarrow(\mathbb{T}, \psi)$; thus, showing that (4) implies (2).

Corollary 6.4. (1) To every infinite minimal circularly ordered cascade $(X, \sigma)$ corresponds a unique (irrational) rotation number $\alpha \in(0,1)$.

(2) Given two minimal circularly ordered cascades $(X, T)$ and $(Y, S)$, they either have rationally independent rotation numbers, in which case they are disjoint; or their rotation numbers are rationally dependent and in this case they admit a common extension of the form $\operatorname{Split}\left(\mathbb{T}, R_{\alpha} ; A\right)$.

6.1. Enveloping semigroups which are c-ordered. We now turn to the study of the enveloping semigroup $E\left(\mathbb{T}, R_{\alpha} ; A\right)$ of $\operatorname{Split}\left(\mathbb{T}, R_{\alpha} ; A\right)$. In [16, Example 14.10] we have shown that when $A$ is a single orbit of the system $\left(\mathbb{T}, R_{\alpha}\right)$, say $A=\{n \alpha: n \in \mathbb{Z}\}$, then $E=$ 
$E\left(\mathbb{T}, R_{\alpha} ; A\right)$ can be identified with the disjoint union $\mathbb{T}_{\mathbb{T}} \cup\left\{\sigma^{n}: n \in \mathbb{Z}\right\}$, where $\left(\mathbb{T}_{\mathbb{T}}, \sigma\right)$ is Ellis' double circle cascade: $\mathbb{T}_{\mathbb{T}}=\left\{\beta^{ \pm}: \beta \in \mathbb{T}=[0,1)\right\}$ and $\sigma \circ \beta^{ \pm}=(\beta+\alpha)^{ \pm}$. E becomes a circularly ordered cascade, where $E=\mathbb{T}_{\mathbb{T}} \cup \mathbb{Z}$ is a c-ordered subset of the c-ordered lexicographic order $\mathbb{T} \times\{-, 0,+\}$ (see Remark 2.12). Under this definition for every $n \in \mathbb{Z}$ we have $\left[n \alpha^{+}, \sigma^{n}, n \alpha^{-}\right]$. Since the interval $\left(n \alpha^{+}, n \alpha^{-}\right) \subset E$ contains only the single element $\sigma^{n}$ for every $n \alpha \in G=\mathbb{Z}$ we get that every element of $G=j(G)$ is isolated in $E$. So, in this case $E=\mathbb{T}_{\mathbb{T}} \cup \mathbb{Z}$, where each point of $\mathbb{Z}$ is isolated in $E$.

Since clearly every $\operatorname{Split}\left(\mathbb{T}, R_{\alpha} ; A\right)$ is a factor of $\mathbb{T}_{\mathbb{T}} \cup \mathbb{Z}$ we have the following.

Corollary 6.5. For every irrational $\alpha \in \mathbb{R}$ and every $R_{\alpha}$-invariant subset $A \subset \mathbb{T}$, the enveloping semigroup of $\operatorname{Split}\left(\mathbb{T}, R_{\alpha} ; A\right)$ is the circularly ordered cascade $\mathbb{T}_{\mathbb{T}} \cup \mathbb{Z}$. It contains $\mathbb{T}_{\mathbb{T}}$ as its unique minimal left ideal; or equivalently as its unique minimal subset.

Proposition 6.6. Every infinite, point transitive, circularly ordered cascade $(Y, S)$ is a factor of $\mathbb{T}_{\mathbb{T}} \cup \mathbb{Z}$ as above.

Proof. We sketch the straightforward proof. If $(Y, S)$ is minimal then by Theorem 6.1 it is of the form $\left(\operatorname{Split}\left(\mathbb{T}, R_{\alpha} ; A\right)\right.$ and we are done. So we now assume that $(Y, S)$ is not minimal.

Suppose $y_{1}, y_{2}, y_{3}$ are three distinct points such that $\left[y_{1}, y_{2}, y_{3}\right]$ and such that the intervals $\left(y_{1}, y_{2}\right)$ and $\left(y_{2}, y_{3}\right)$ are empty. Then $y_{2}$ is an isolated point and is therefore necessarily on the unique orbit of transitive points.

Let $\Omega$ be the smallest ICER which contains the pairs $\left(y, y^{\prime}\right) \in Y \times Y$ such that the interval $\left(y, y^{\prime}\right)$ is empty. It is not hard to see, using the remark above, that $Y / \Omega$ is a circle on which $S$ induces a minimal homeomorphism. Then $Y / \Omega$ can be identified with $\left(\mathbb{T}, R_{\alpha}\right)$ for some irrational $\alpha$ and one checks that the quotient map $\pi:(Y, S) \rightarrow\left(\mathbb{T}, R_{\alpha}\right)$ is a proximal extension.

This implies that $Y$ contains a unique minimal subset $Z \subset Y$, and by Theorem 6.1 $Z \cong \operatorname{Split}\left(\mathbb{T}, R_{\alpha}, A\right)$ for some irrational $\alpha$ and an $R_{\alpha}$-invariant $A \subset \mathbb{T}$.

Next one shows that for every $z \in A$ the corresponding open interval $\left(z^{-}, z^{+}\right)$contains at most one point and moreover, there is a unique orbit $\{z+n \alpha: n \in \mathbb{Z}\} \subset A$ for which this happens; i.e. there are points $c_{n}$ with $\left[(z+n \alpha)^{-}, c_{n},(z+\alpha)^{+}\right]$. We now identify $c_{n}$ with $S^{n}$. Finally, using the information we already have on $(Y, S)$ it is clear how to define the factor map $\mathbb{T}_{\mathbb{T}} \cup \mathbb{Z} \rightarrow(Y, S)$.

6.2. General discrete group action. Let $G$ be an infinite countable group. A $G$-system $(G, X)$ is proximal if for every pair $x, x^{\prime} \in X$ and a every neighborhood $V$ in $X \times X$ of the diagonal $\Delta_{X}=\{(z, z): z \in X\}$, there is $g \in G$ with $\left(g x, g x^{\prime}\right) \in V$. We say that $(G, X)$ is extremely proximal if for every closed subset $A \subset X$ and every nonempty open subset $U \subset X$ there exists an element $g \in G$ with $g(A) \subset U$. Recall that the $G$-action on a metrizable $X$ is equicontinuous iff there is a compatible metric on $G$ with respect to which every $g \in G$ acts as an isometry. We then say that the action is isometric.

We have the following neat dichotomy theorem of Malyutin [31]:

Theorem 6.7. Every minimal system $(G, \mathbb{T})$, a continuous action of $G$ on the circle $\mathbb{T}$, is either conjugate to an isometric action on $\mathbb{T}$ or it is a finite to one extension of an extremely proximal action where the factor map is a covering map.

It is easy to see that a minimal proximal $G$-action of an abelian group $G$ is necessarily trivial. Thus it follows that every minimal action of abelian $G$ on $\mathbb{T}$ is isometric. An example of a minimal proximal action is provided by the natural action of $S L(2, \mathbb{Z})$ on the projective line $\mathbb{P}^{1}$, the collection of lines through the origin in $\mathbb{R}^{2}$ (which is homeomorphic to $\mathbb{T})$. The action of $S L(2, \mathbb{Z})$ on the space of rays emanating from the origin, which is again homeomorphic to $\mathbb{T}$, is an example of a two-to-one covering of a proximal action. 
Theorem 6.8. Let $(G, X)$ be an infinite circularly ordered minimal $G$-system. Then there exists a minimal $G$-action on $\mathbb{T}$ and a $G$-invariant subset $A \subset \mathbb{T}$ such that: $(G, X) \cong$ $\operatorname{Split}(\mathbb{T}, G ; A)$.

Proof. Same as the (relevant parts of the) proof of Theorem 6.1.

\section{REFERENCES}

1. E. Akin, K. Hrbacek, Complete homogeneous LOTS, Topology Proc. 26 (2001), 367-406.

2. J. B. Aujogue, Ellis enveloping semigroup for almost canonical model sets, ArXiv:1305.0879, May, 2014.

3. J. Bourgain, D.H. Fremlin and M. Talagrand, Pointwise compact sets in Baire-measurable functions, Amer. J. of Math., 100:4 (1977), 845-886.

4. V. Berthe, S. Ferenczi, L.Q. Zamboni, Interactions between Dynamics, Arithmetics and Combinatorics: the Good, the Bad, and the Ugly, Contemporary Math., 385 (2005), 333-364.

5. E. Čech, Point Sets, Academia, Prague, 1969.

6. S. Černak, J. Jakubik, Completion of a cyclically ordered group, Czech. Math. J., 37 (1987), 157-174.

7. A. Chernikov and P. Simon, Definably amenable NIP groups, arXiv:1502.04365v1 [math.LO] 15 Feb 2015.

8. A. Denjoy, Sur les courbes définies par les équations différentielles à la surface du tore, J. de Math. 11, (1932), 333-75.

9. D. van Dulst, Characterizations of Banach spaces not containing $l^{1}$. Centrum voor Wiskunde en Informatica, Amsterdam, 1989.

10. R. Ellis, Lectures on Topological Dynamics, W. A. Benjamin, Inc., New York, 1969.

11. R. Ellis and W. H. Gottschalk, Homomorphisms of transformation groups, Trans. Amer. Math. Soc. 94, (1960), 258-71.

12. T. Fernique, Multi-dimensional Sturmian sequences and generalized substitutions, Int. J. Found. Comput. Sci., 17 (2006), pp. 575-600.

13. E. Glasner, Proximal flows, Lect. Notes, 517, Springer, 1976.

14. E. Glasner, On tame dynamical systems, Colloq. Math. 105 (2006), 283-295.

15. E. Glasner, The structure of tame minimal dynamical systems, Ergod. Th. and Dynam. Sys. 27 (2007), 1819-1837.

16. E. Glasner, M. Megrelishvili, Linear representations of hereditarily non-sensitive dynamical systems, Colloq. Math., 104 (2006), no. 2, 223-283.

17. E. Glasner and M. Megrelishvili, New algebras of functions on topological groups arising from G-spaces, Fundamenta Math., 201 (2008), 1-51.

18. E. Glasner, M. Megrelishvili, Representations of dynamical systems on Banach spaces not containing $l_{1}$, Trans. Amer. Math. Soc., 364 (2012), 6395-6424. ArXiv e-print: 0803.2320.

19. E. Glasner, M. Megrelishvili, Banach representations and affine compactifications of dynamical systems, in: Fields institute proceedings dedicated to the 2010 thematic program on asymptotic geometric analysis, M. Ludwig, V.D. Milman, V. Pestov, N. Tomczak-Jaegermann (Editors), Springer, New-York, 2013. ArXiv version: 1204.0432 .

20. E. Glasner, M. Megrelishvili, Representations of dynamical systems on Banach spaces, in: Recent Progress in General Topology III, (Eds.: K.P. Hart, J. van Mill, P. Simon), Springer-Verlag, Atlantis Press, 2014, 399-470.

21. E. Glasner, M. Megrelishvili, Eventual nonsensitivity and tame dynamical systems, arXiv:1405.2588, 2014.

22. E. Glasner, M. Megrelishvili and V.V. Uspenskij, On metrizable enveloping semigroups, Israel J. of Math. 164 (2008), 317-332.

23. W. Huang, Tame systems and scrambled pairs under an abelian group action, Ergod. Th. Dynam. Sys. 26 (2006), 1549-1567.

24. E.V. Huntington, Sets of completely independent postulates for cyclic order, Proc. Nat. Acad. Sci. USA 10 (1924). 74-78.

25. T. Ibarlucia, The dynamical hierarchy for Roelcke precompact Polish groups, Israel J. of Math., to appear.

26. A.S. Kechris, V.G. Pestov, and S. Todorčević, Fraïssé limits, Ramsey theory, and topological dynamics of automorphism groups, Geom. Funct. Anal. 15 (2005), no. 1, 106-189.

27. D. Kerr and H. Li, Dynamical entropy in Banach spaces, Invent. Math. 162 (2005), 649-686.

28. D. Kerr and H. Li, Independence in topological and $C^{*}$-dynamics, Math. Ann. 338 (2007), 869-926.

29. H. Kok, Connected orderable spaces, Math. Centhre Tracts 49, Mathematisch Centrum, Amsterdam, 1973. 
30. A. Köhler, Enveloping semigrops for flows, Proc. of the Royal Irish Academy, 95A (1995), $179-191$.

31. A. V. Malyutin, Classification of the group actions on the real line and circle, Algebra i analiz, Tom 19, (2007), N. 2. St. Petersburg Math. J. Vol 19 (2008), No. 2, 279-296.

32. N. G. Markley, Homeomorphisms of the circle without periodic points, Proc. London Math. Soc, (3) 20, (1970), 688-698.

33. G. Martinez-Cervantes, On weakly Radon-Nikodym compact spaces, ArXiv 1509.05324, September, 2015.

34. K. Masui, Denjoy systems and substitutions, Tokyo J. Math. 32:1 (2009), 33-53.

35. M. Megrelishvili, A note on tameness of families having bounded variation, ArXiv, 2014.

36. M. Morse, G. A. Hedlund, Symbolic Dynamics II. Sturmian Trajectories, American J. of Math., Vol. 62, No. 1 (1940), pp. 1-42.

37. L. Nachbin, Topology and order, Van Nostrand Math. Studies, Princeton, New Jersey, 1965.

38. I. Namioka, Radon-Nikodým compact spaces and fragmentability, Mathematika 34 (1987), 258-281.

39. R. Pikula, Enveloping semigroups of affine skew products and Sturmian-like systems, Dissertation, The Ohio State University, 2009.

40. V.G. Pestov, On free actions, minimal flows, and a problem by Ellis, Trans. Amer. Math. Soc., 350 (1998), 4149-4165.

41. V.G. Pestov, Dynamics of infinite-dimensional groups. The Ramsey-Dvoretzky-Milman phenomenon. University Lecture Series, 40. American Mathematical Society, Providence, RI, 2006.

42. H. Poincaré, Sur les courbes définies par les équations différentielles, J. Math. Pures Appl. (4), 1, (1885), 167-224.

43. H.P. Rosenthal, A characterization of Banach spaces containing $l_{1}$, Proc. Nat. Acad. Sci. U.S.A., 71 (1974), 2411-2413.

44. A.V. Romanov, Ergodic properties of discrete dynamical systems and enveloping semigroups, Ergod. Th. \& Dynam. Sys. 36 (2016), 198-214.

45. M. Talagrand, Pettis integral and measure theory, Mem. AMS No. 51, 1984.

46. S. Todorčević, Topics in topology, Lecture Notes in Mathematics, 1652, Springer-Verlag, 1997.

47. V.V. Uspenskij, Compactifications of topological groups, Proceedings of the Ninth Prague Topological Symposium (Prague, August 19-25, 2001). Edited by P. Simon. Published April 2002 by Topology Atlas (electronic publication). Pp. 331-346, ArXiv:math.GN/0204144.

48. L. Nguyen van Thé, More on the Kechris-Pestov-Todorcevic correspondence: precompact expansions, Arxiv: 1201.1270v3.

Department of Mathematics, Tel-Aviv University, Ramat Aviv, Israel

E-mail address: glasner@math.tau.ac.il

$U R L:$ http: //www.math.tau.ac.il/ glasner

Department of Mathematics, Bar-Ilan University, 52900 Ramat-Gan, IsRael

E-mail address: megereli@math.biu.ac.il

URL: http://www.math.biu.ac.il/ megereli 